\title{
Fabrication of NIS and SIS Nanojunctions with Aluminum Electrodes and Studies of Magnetic Field Influence on IV Curves
}

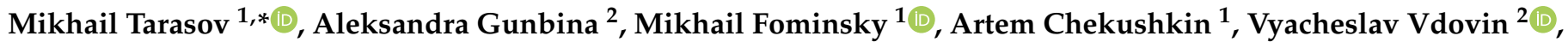 \\ Valery Koshelets ${ }^{1}\left[\right.$, Elizaveta Sohina ${ }^{3,4}$, Alexei Kalaboukhov ${ }^{5}\left(\mathbb{D}\right.$ and Valerian Edelman ${ }^{3}$ \\ 1 V. Kotelnikov Institute of Radio Engineering and Electronics of RAS, 103907 Moscow, Russia; \\ demiurge@hitech.cplire.ru (M.F.); chekushkin@hitech.cplire.ru (A.C.); valery@hitech.cplire.ru (V.K.) \\ 2 Institute of Applied Physics of RAS, 603155 Nizhny Novgorod, Russia; aleksandragunbina@mail.ru (A.G.); \\ vdovin@ipfran.ru (V.V.) \\ 3 P.L. Kapitza Institute for Physical Problems, 119334 Moscow, Russia; eliza-so@yandex.ru (E.S.); \\ vsedelman@yandex.ru (V.E.) \\ 4 NIU Higher School of Economics, 109028 Moscow, Russia \\ 5 Department Quantum Device Physics, Chalmers University of Technology, SE41296 Göteborg, Sweden; \\ alexei.kalaboukhov@chalmers.se \\ * Correspondence: tarasov@hitech.cplire.ru
}

\section{check for} updates

Citation: Tarasov, M.; Gunbina, A.; Fominsky, M.; Chekushkin, A.; Vdovin, V.; Koshelets, V.; Sohina, E.; Kalaboukhov, A.; Edelman, V. Fabrication of NIS and SIS Nanojunctions with Aluminum Electrodes and Studies of Magnetic Field Influence on IV Curves. Electronics 2021, 10, 2894. https:/ / doi.org/10.3390/electronics10232894

Academic Editor: Lingwei Li

Received: 15 October 2021

Accepted: 22 November 2021

Published: 23 November 2021

Publisher's Note: MDPI stays neutral with regard to jurisdictional claims in published maps and institutional affiliations.

Copyright: (c) 2021 by the authors. Licensee MDPI, Basel, Switzerland. This article is an open access article distributed under the terms and conditions of the Creative Commons Attribution (CC BY) license (https:// creativecommons.org/licenses/by/ $4.0 /)$.
Abstract: Samples of superconductor-insulator-superconductor (SIS) and normal metal-insulatorsuperconductor (NIS) junctions with superconducting aluminum of different thickness were fabricated and experimentally studied, starting from conventional shadow evaporation with a suspended resist bridge. We also developed alternative fabrication by magnetron sputtering with two-step direct e-beam patterning. We compared Al film grain size, surface roughness, resistivity deposited by thermal evaporation and magnetron sputtering. The best-quality NIS junctions with large superconducting electrodes approached a resistance $R(0) / R\left(V_{2 \Delta}\right)$ factor ratio of 1000 at $0.3 \mathrm{~K}$ and over 10,000 at $0.1 \mathrm{~K}$. At $0.1 \mathrm{~K}, \mathrm{R}(0)$ was determined completely by the Andreev current. The contribution of the single-electron current dominated at $\mathrm{V}>\mathrm{V}_{\Delta / 2}$. The single-electron resistance extrapolated to $\mathrm{V}=0$ exceeded the resistance $\mathrm{R}\left(\mathrm{V}_{2 \Delta}\right)$ by $3 \times 10^{9}$. We measured the influence of the magnetic field on NIS junctions and described the mechanism of additional conductivity due to induced Abrikosov vortices. The modified shape of the SINIS bolometer IV curve was explained by Joule overheating via NIN (normal metal-insulator-normal metal) channels.

Keywords: SIS (superconductor-insulator-superconductor) junctions; SIN (superconductor-insulatornormal metal) junctions; Abrikosov vortices

\section{Introduction}

To date, the common material for superconducting electronics is Niobium and its nitrides, $\mathrm{NbN}$ and $\mathrm{NbTiN}$. The main reasons for this are that $\mathrm{Nb}$-based devices can operate at liquid helium temperature, and due to well-developed and reproducible technology with magnetron sputtering. The drawback of $\mathrm{Nb}$ technology is that it is very sensitive to contamination, which can suppress the transition temperature and quality of junctions, the granular structure of films, and the presence of Abrikosov's vortices inevitable for secondtype superconductors. With the progress in qubit, the quantum computer, and singlephoton detectors, the operation temperature of high-performance superconducting devices reduces to well below $1 \mathrm{~K}$ and allows the use of aluminum as a superconducting electrode. Contrary to $\mathrm{Nb}$, in $\mathrm{Al}$, film contaminations can provide fine tuning of the transition temperature from $1.2 \mathrm{~K}$ in pure bulk to the range of 0.1 to $2.4 \mathrm{~K}$ [1]. This is the first-type superconductor, and for relatively thick films, there are no penetrated vortices, associated flux pinning/depinning, or accompanied excess noise, contrary to thin aluminum films 
below $100 \mathrm{~nm}$, which can demonstrate some features of superconductor type-2 [2]. For films with thicknesses over $5 \mathrm{~nm}$, the critical temperature monotonically decreases with a decrease in film resistance per square [3]. Thin aluminum films can be rather smooth with less defects and better quality of tunnel barriers. Deposition can be performed by thermal evaporation, as well as magnetron sputtering. Deposition and patterning methods can be very flexible and reproducible. On the other hand, thin aluminum film in the magnetic field can show some features of the type-2 superconductor with flux pinning, additional losses, current leak, and overheating in tunnel junctions. We investigated possible mechanisms of losses and the influence of magnetic field on the IV curves of NIS junctions in the temperature range $0.08-0.35 \mathrm{~K}$. The presence of an additional conductivity mechanism via Andreev reflection makes the IV curve more complicated compared to single-electron tunneling in ideal NIS junctions.

\section{Fabrication}

For the fabrication of aluminum tunnel junctions, we used three different technologies: Dolan's technique [4], bridge-free technology [5,6] and direct write technology with magnetron sputtering [7]. The description and comparison of such technologies are presented in [8].

To avoid magnetic flux penetration, the first obvious decision is increasing film thickness to over $\xi_{0}$ or even $\lambda$, i.e., $15-100 \mathrm{~nm}$. However, such increase can be accompanied with an increase in grain size, and grain boundaries can become traps for frozen vortices. Reducing the grain size or making the film surface flatter can be achieved by producing epitaxial films on an appropriate substrate with a lattice period close to that of aluminum. Surface roughness is a good indicator of film quality. In the following, we compare several different films deposited by thermal evaporation from resistive boat [1], electron beam evaporation (Figure 1), and magnetron sputtering (Figure 2). Morphological analysis using an Atomic Force Microscope (AFM) was carried out through a Bruker Dimension Icon AFM, equipped with a Nanoscope $\mathrm{V}$ controller operated in PeakForce mode. The measurements were performed using N-doped Si probes (Bruker Scanasyst-air).

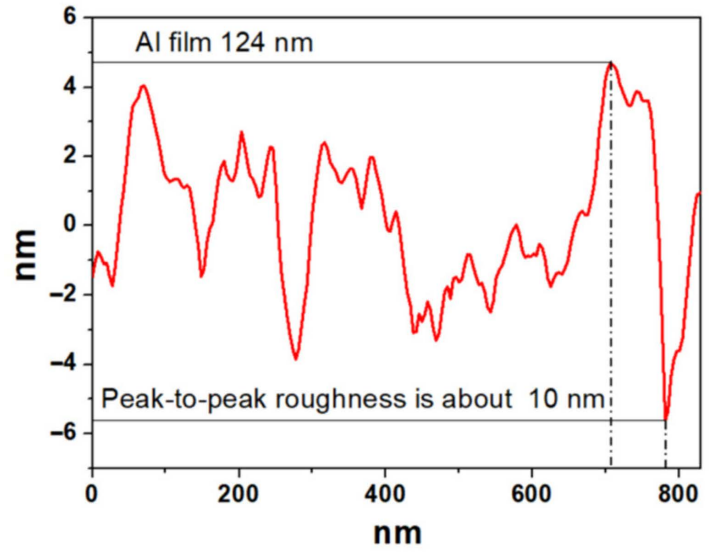

(a)

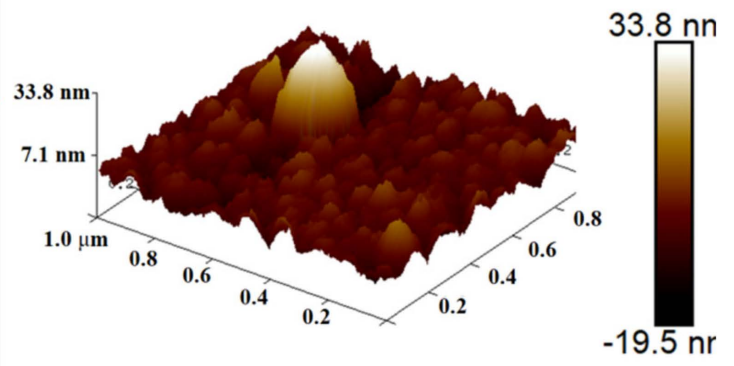

(b)

Figure 1. (a) Surface profile for $\mathrm{Al}$ film of $124 \mathrm{~nm}$ thick; peak-to-peak roughness is approximately $\pm 5 \mathrm{~nm}$. (b) AFM image. Lateral grain size is comparable to film thickness. The largest grain in $3 \mathrm{~d}$ view is $40 \mathrm{~nm}$ high. Al films were fabricated by electron beam evaporation.

For 20 and $6 \mathrm{~nm}$-thick films prepared by thermal evaporation, the peak-to-peak roughness was \pm 2 and $\pm 0.5 \mathrm{~nm}$ [1]. If we take into account that the $\mathrm{Al}_{2} \mathrm{O}_{3}$ tunnel barrier thickness for our junctions of $1 \mathrm{k} \Omega \times \mu \mathrm{m}^{2}$ was approximately $1.8 \mathrm{~nm}$, it means that a higher quality of NIS junctions can be obtained with thin films of absorbers below $10 \mathrm{~nm}$. The AFM analysis of Al films of 3 and $6 \mathrm{~nm}$ thick deposited by magnetron sputtering is presented in Figure 2. The top layer of superconducting aluminum should be over 
$150 \mathrm{~nm}$ (Figure 3a). The best-quality NIS junctions with large and thick superconducting electrodes approach resistance $R(0) / R\left(V_{2 \Delta}\right)$ factor ratio of 1000 at $0.3 \mathrm{~K}$ and over 10,000 at $0.1 \mathrm{~K}$ (Figure 3b).
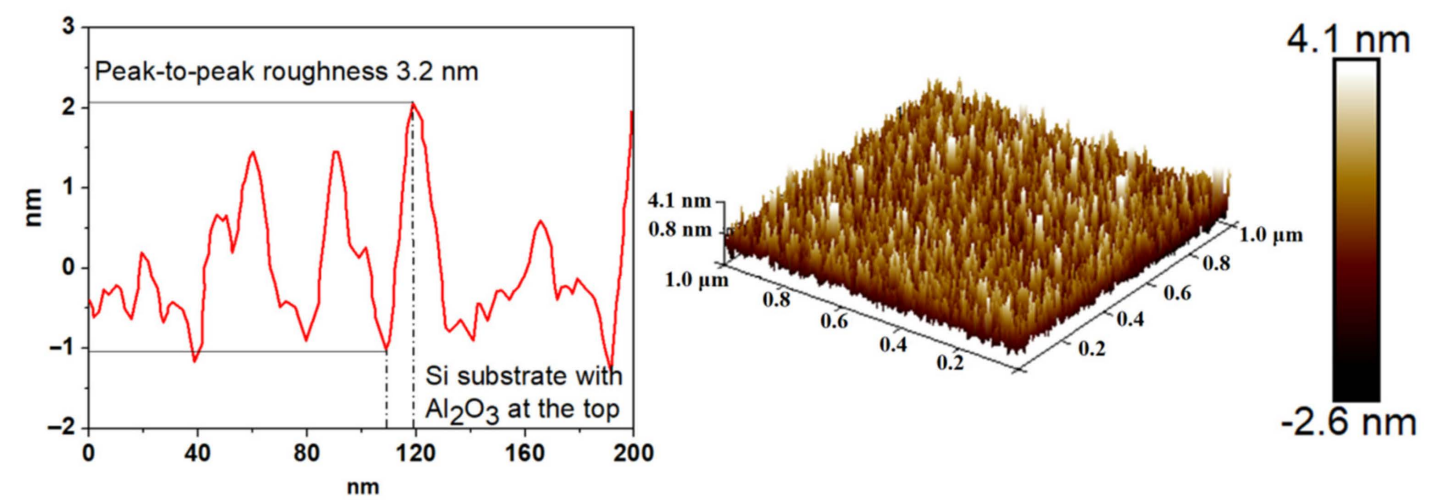

(a)
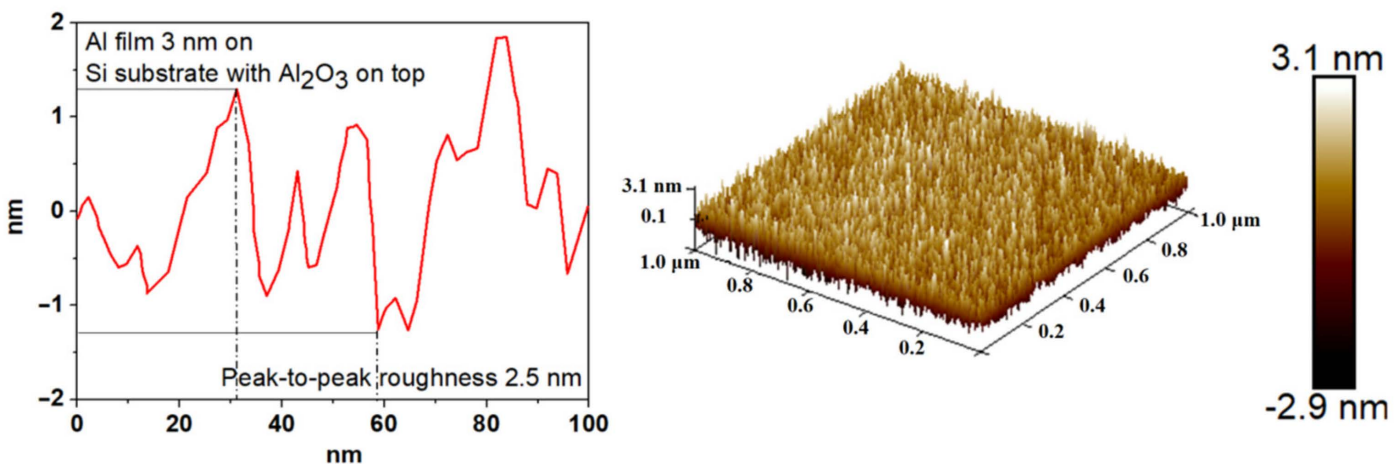

(b)
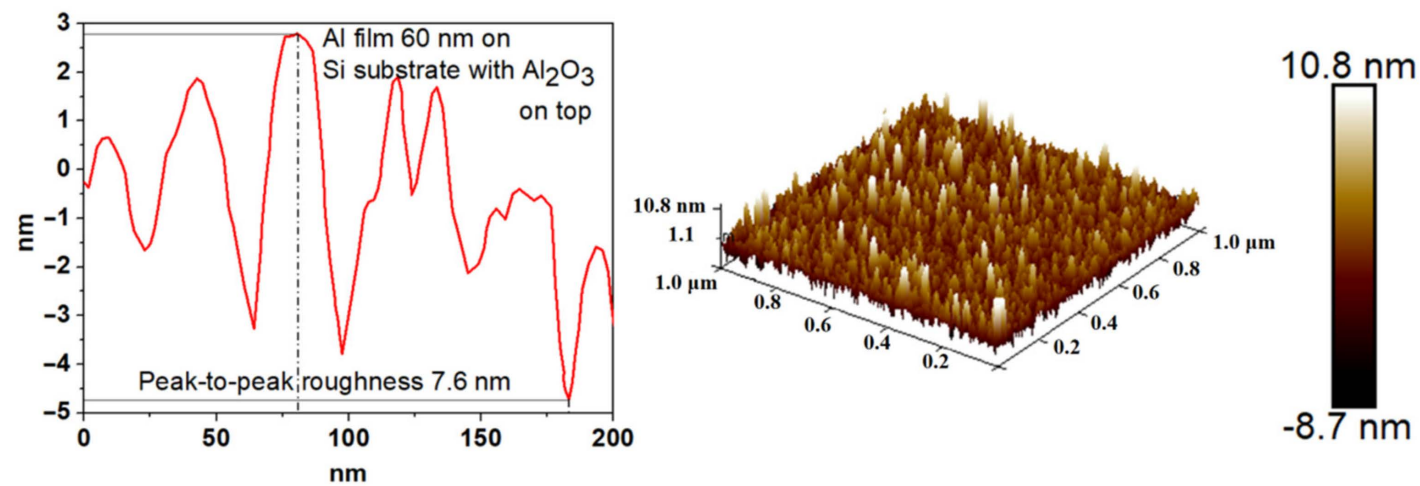

(c)

Figure 2. (a) Si substrate with $\mathrm{Al}_{2} \mathrm{O}_{3}$ at the top. Profile of this substrate. Peak-to-peak roughness of $3.2 \mathrm{~nm}$; (b) Al film of $3 \mathrm{~nm}$ on the top of this substrate. Profile of this film. Peak-to-peak roughness of $2.5 \mathrm{~nm}$ (less compared to substrate); (c) Al film of $6 \mathrm{~nm}$ on similar substrate. Profile of this film; peak-to-peak roughness of $7.6 \mathrm{~nm} . \mathrm{Al}_{\text {and }} \mathrm{Al}_{2} \mathrm{O}_{3}$ films were fabricated by magnetron sputtering.

The comparison of peak-to-peak roughness and average grain width for films of different thickness fabricated by three methods is presented in Table 1.

The overall features of films are not very much dependent on substrate roughness and fabrication method. For films $20 \mathrm{~nm}$ and thinner peak-to-peak roughness is about a half of thickness, grain size about twice as much. For 60 and $124 \mathrm{~nm}$ films p/p roughness is $10 \mathrm{~nm}$ and below, grain size close to the thickness. Films $150 \mathrm{~nm}$ and thicker are smoother with roughness on average $5 \mathrm{~nm}$ and below and grain size about $500 \mathrm{~nm}$. Such behavior in some way is correlated with critical temperature variations that is $1.2 \mathrm{~K}$ for thick films and 
can increase up to about $1.9 \mathrm{~K}$ for thin films. Possible mechanisms of superconductivity in such films will be studied by measurements of IV curves of tunnel junctions fabricated of such films. We also fabricated SIS junctions with the similar layout $[8,9]$. The difference in SIS fabrication is that only $\mathrm{Al}$ is deposited as the first layer, contrary to NIS junctions for which the underlayer of 1-2 $\mathrm{nm}$ of Fe was deposited before Al. Influence of magnetic field on SIS junction is more complicated, including oscillations of Josephson critical current of SIS junction and penetration of Abrikosov vortices in lower Al layer, as well as in upper $\mathrm{Al}$ film. Contrary to SIS, in NIS junctions we have only one effect of vortices in upper Al film that makes explanation clear and unambiguous.

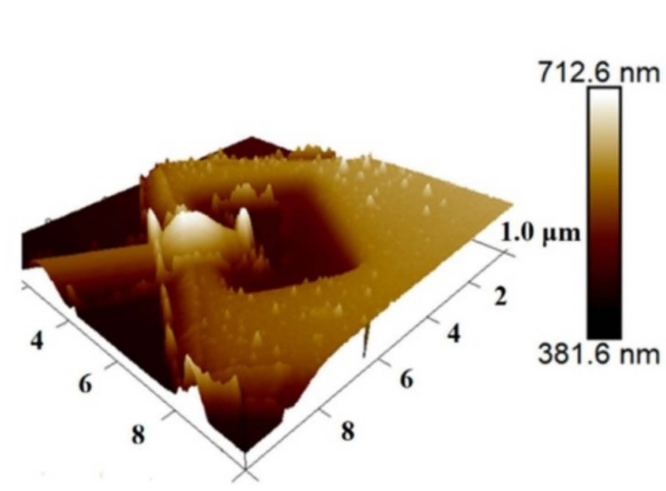

(a)

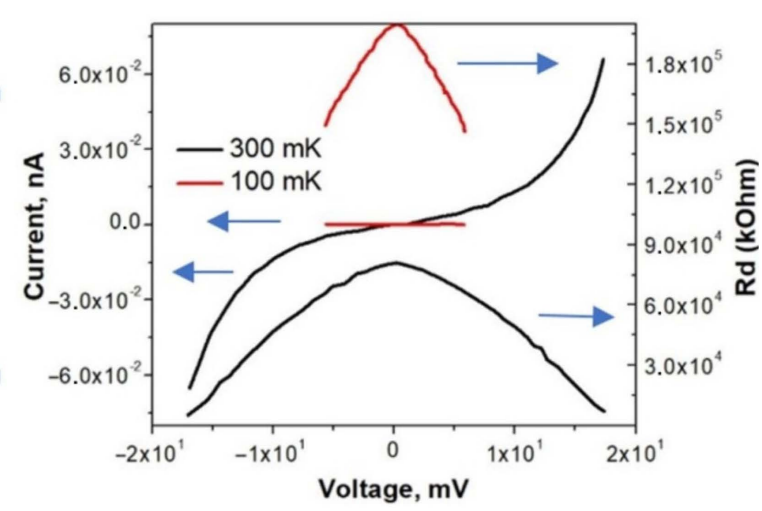

(b)

Figure 3. (a) Al NIS junction with 150 nm-thick aluminum electrode fabricated by magnetron sputtering; (b) IV curves and dynamic resistance for this NIS junction. Peak-to-peak roughness in the region out of peaks is $5 \mathrm{~nm}$, and grain size is $400 \mathrm{~nm}$.

Table 1. Film thickness and surface features.

\begin{tabular}{cccc}
\hline Method & Thickness, $\mathbf{n m}$ & Roughness $\mathbf{p} / \mathbf{p}, \mathbf{n m}$ & Grain Width, $\mathbf{n m}$ \\
\hline Hot boat & 3 & 1.8 & 8 \\
Hot boat & 6 & 3.4 & 18 \\
Hot boat & 20 & 6.7 & 40 \\
El. beam & 3 & 2.5 & 10 \\
El. beam & 60 & 7.6 & 70 \\
El. beam & 124 & 10 & 100 \\
Magnetron & 150 & 5 & 400 \\
\hline
\end{tabular}

\section{Influence of Permanent Magnetic Field on Conductivity of NIS Junctions}

To characterize the obtained aluminum films, it is important to study them in a magnetic field. This makes it possible to judge how much their properties correspond to those of bulk aluminum. Pure thick aluminum is a type- 1 conventional superconductor with a critical temperature of $T_{c}=1.2 \mathrm{~K}$, critical field of $\mathrm{B}_{\mathrm{c}}=11 \mathrm{mT}$, coherence length of $\xi_{0}=1.5 \mu \mathrm{m}$, and magnetic field penetration depth of $\lambda_{0}=15 \mathrm{~nm}$. The grain structure of actual thin films depends on the film thickness and deposition method, in addition to potential contamination. Earlier in our experiments, in the magnetic field normal to the film plane, a nonlinearity at up to $\sim 30 \mathrm{mT}$ was observed, which is above the critical field for bulk aluminum [2]. This means that $\mathrm{Al}$ films of $80 \mathrm{~nm}$ thick are not type-1 superconductors.

In our experiments, the influence of the magnetic field was observed for single NIS, as well as for SINIS structures, including those integrated into planar antennas. In all cases, at temperatures of $0.1-0.3 \mathrm{~K}$ in fields of $10-30 \mathrm{mT}$ at low bias voltages, an increase in the conductivity from tens to hundreds of times was observed. However, the analysis of the results in these cases is complicated by their noticeable overheating by parasitic background thermal radiation from the environment. The most immune to external noise and interferences are the multijunction NIS thermometers with 100 elements connected in 
series. We studied in detail the influence of magnetic field on the multijunction NIS thermometer. It contains unit cells of five parallel-connected identic NIS junctions connected in series of $n=100$ such parallel cells. A single junction in the thermometer comprised an aluminum electrode covering an oxidized normal electrode made of aluminum with an Fe underlayer for the suppression of superconductivity. Dimensions of the tunnel junction are $S_{\text {NIS }}=1.8 \times 1 \mu \mathrm{m}^{2}$, and aluminum thickness is $80 \mathrm{~nm}$. Each junction is connected to Au wiring film of $14 \times 100 \times 0.1=140 \mu \mathrm{m}^{3}$. During the measurements, the structures were cooled down to a temperature of $\sim 0.1 \mathrm{~K}$ in a portable dilution cryostat [10]. The temperature was measured with a resistance thermometer based on a planar RuO resistor calibrated by a paramagnetic salt thermometer. The IV curves were measured under computer control in the current bias mode set by a 16-bit DAC. The measured voltage was amplified and digitized with a 16-bit ADC. The IV curve at $0.096 \mathrm{~K}$ is presented in Figure 4. A constant magnetic field perpendicular to the substrate was created by a solenoid located outside the cryostat. The superconducting gap $\Delta_{\mathrm{c}} / \mathrm{k}=2.07 \mathrm{~K}\left(\mathrm{~V}_{\Delta}=\Delta_{\mathrm{c}} / \mathrm{e}=178 \mu \mathrm{V}\right)$ and $R_{n}=9 \mathrm{k} \Omega$ were estimated from dynamic resistance measured at a temperature in the range $0.4-0.1 \mathrm{~K}$.

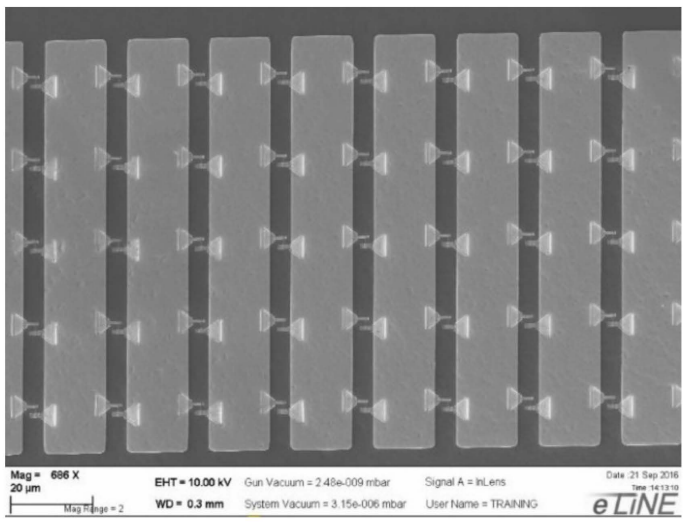

(a)

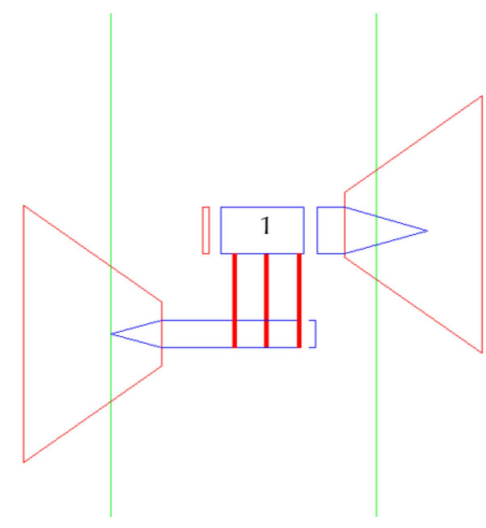

(b)

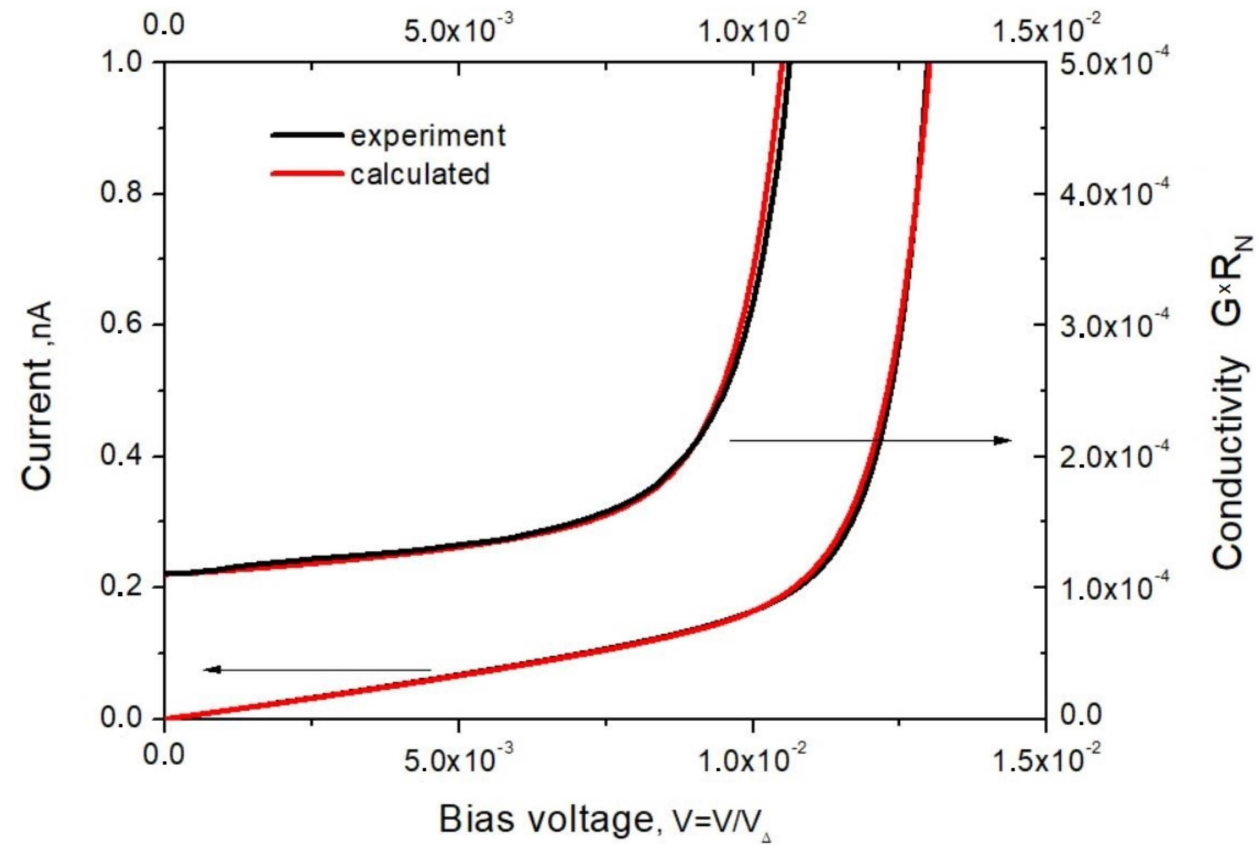

(c)

Figure 4. Photo of a fraction of multijunction NIS array thermometer (a), design of NIS junction 1 of the area $1 \times 1.8 \mu \mathrm{m}^{2}(\mathbf{b})$, IV curves and relative dynamic conductivity, experimental and theoretical curves including both Andreev's current (1) and single-electron current (c). 
A specific feature of the IV curves is the large impact of the subgap current at bias voltages below $0.5 \times \mathrm{n} \times \Delta_{\mathrm{c}}$. This can be attributed to Andreev's current, which can be described according to [11] by the expression for $n$ junctions in series:

$$
\mathrm{I}_{\mathrm{a}}=\mathrm{K}_{\mathrm{N}} \tanh \left(\frac{\mathrm{eV}}{2 \mathrm{nkT}_{\text {eff }}}\right)+\mathrm{K}_{\mathrm{S}} \frac{\mathrm{V}}{\sqrt{1-\frac{\mathrm{V}}{\mathrm{V}_{\Delta / \mathrm{n}}}}}
$$

with three fitting parameters that should be selected from experiment [12]. An alternative approach is the Dynes model [13]. Initially, it was suggested for describing IV curves at bias voltages around the energy gap $\Delta_{\mathrm{c}} / \mathrm{e}$. Later, it was also applied to low-voltage regions. In this model, the subgap current is presented as follows:

$$
\mathrm{I}_{\mathrm{Dy}}=\frac{\gamma}{\mathrm{R}_{\mathrm{N}}} \frac{\mathrm{V}}{\sqrt{1-\left(\frac{\mathrm{V}}{\mathrm{V}_{\Delta / \mathrm{n}}}\right)^{2}}}
$$

Contrary to this Dynes model, in (1), the dynamic resistance is linearly dependent on bias voltage at a bias of approximately $\mathrm{V}=0$, which corresponds to our experimental results (Figure 4). For this reason, we prefer to use (1) for describing our IV curves.

The variation of conductivity in the normal to surface magnetic field is presented in Figures 5 and 6 in logarithmic and linear scale. Relative conductivity $G \times R_{n}$ at zero bias and $\mathrm{B}=2.33 \mathrm{mT}$ within noise accuracy is unchanged. Changes in single-electron conductivity can be estimated by differentiating IV curves and subtracting the impact of Andreev's current. According to [14] for pure NIS junction at low temperature and low bias the single-electron tunneling current $\mathrm{I}_{\text {sing }}$ can be presented in common simple form as

$$
\mathrm{I}_{\text {sing }}=\frac{\sqrt{2 \pi \Delta \mathrm{kT}}}{2 \mathrm{e} \mathrm{R}_{\mathrm{n}}} \exp \left[-\frac{\Delta-\mathrm{eV}}{\mathrm{kT}}\right]
$$

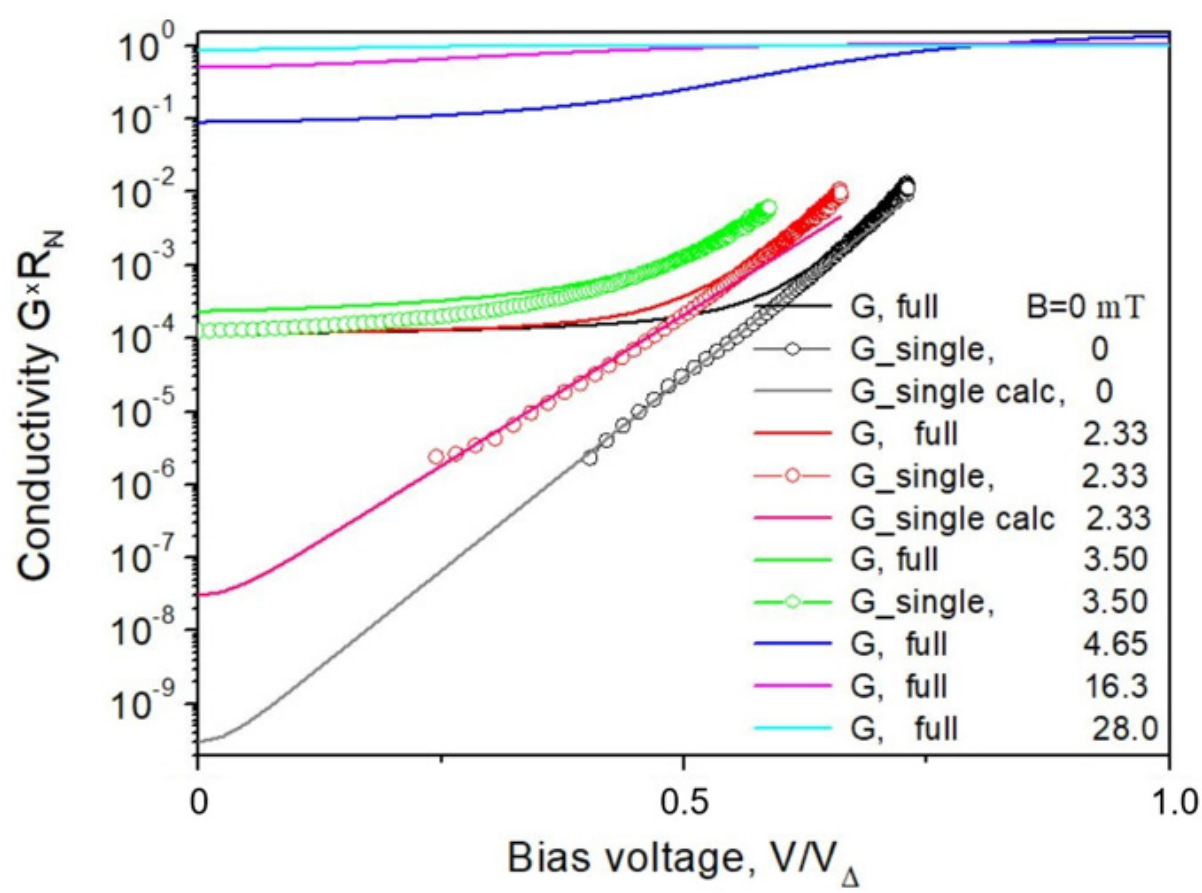

Figure 5. Normalized conductivity $G \times R_{n}$ in logarithmic scale dependence (experimental with circles and calculated lines) on normalized voltage $\mathrm{V} / \mathrm{V}_{\Delta}$ for magnetic field from 0 to $28 \mathrm{mT}$. Curves with label "single" correspond to single-electron tunneling with subtracted Andreev current, and label "full" corresponds to full current including both Andreev conductivity and single-electron tunneling. 


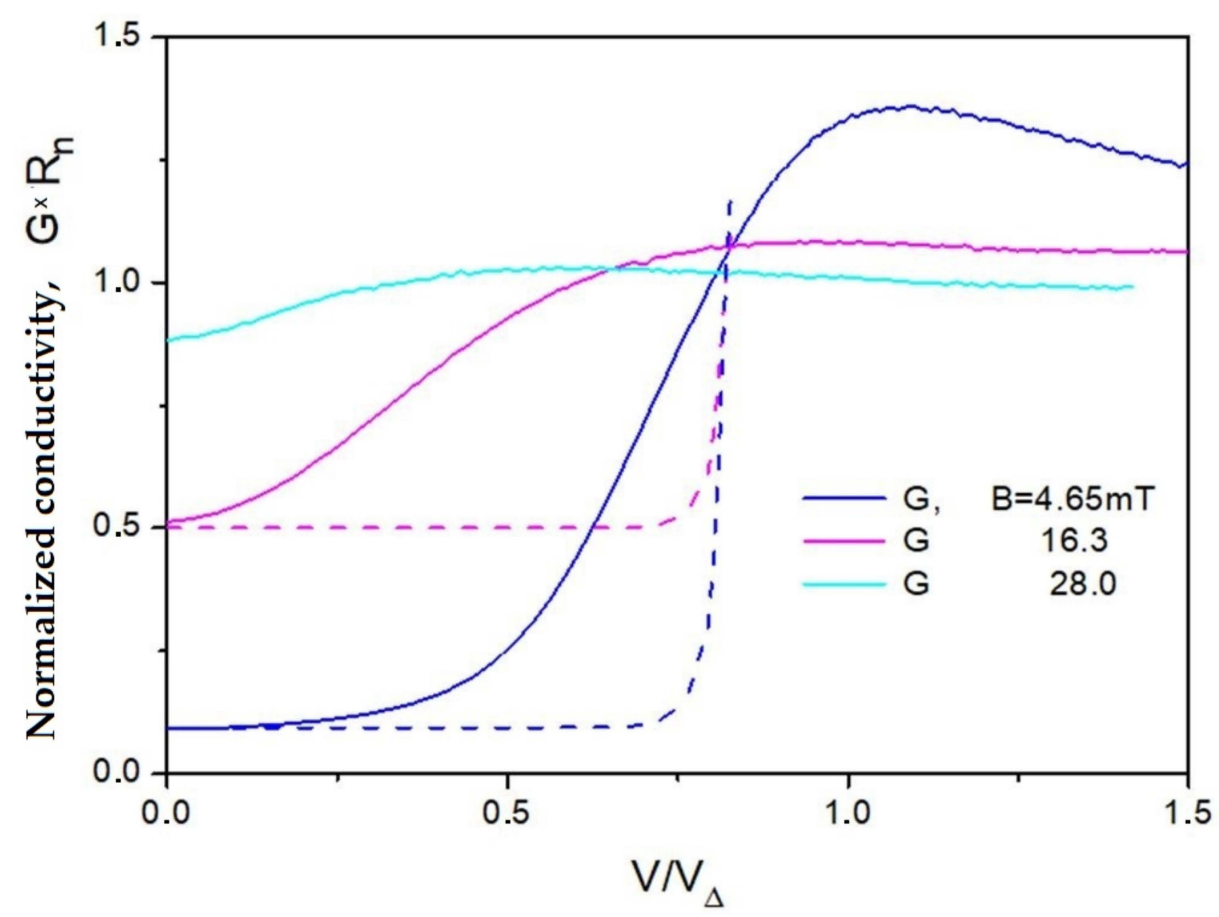

Figure 6. Normalized conductivity $G \times R_{n}$ dependence in linear scale on normalized voltage $V / V_{\Delta}$ for magnetic field of $4.65,16.3$, and $28 \mathrm{mT}$. Dashed lines are simulated for shunting of perfect NIS junction by $0.5 / R_{n}$ and $0.1 / R_{n}$ conductivity.

Or more exactly, according to [15] as

$$
\mathrm{I}_{\text {sing }}=\frac{\sqrt{2 \pi \Delta \mathrm{kT}}}{\mathrm{eR}_{\mathrm{n}}} \exp \left(-\frac{\Delta}{\mathrm{kT}}\right) \sinh \left(\frac{\mathrm{eV}}{\mathrm{kT}}\right)
$$

From Figure 5, it is clear that the magnetic field leads to a reduction in single-electron conductivity by two orders of magnitude, but it is still rather small, at the level of approximately $3 \times 10^{-8}$. When cooling in magnetic field of $3.5 \mathrm{mT}$, the variation is more pronounced; single-electron conductivity increases up to $10^{-4}$, and at $4.65 \mathrm{mT}$, up to 0.09 . For a further increase in the magnetic field, relative conductivity approaches 1 . However, for a higher magnetic field of $28 \mathrm{mT}$, the conductivity is still slightly below unity, nonlinearity and superconductivity are still present, which means that although the critical field is not achieved but is very close to it. Therefore, to estimate the correlation length with an accuracy of $\sim 10 \%$, we can use the value of $30 \mathrm{mT}$ as a critical field $\mathrm{B}_{\mathrm{C} 2}$.

Pure aluminum is a conventional type- 1 superconductor. The grain structure of actual thin films depends on the film thickness and deposition method, in addition to potential contamination. As a result, such films can be similar to type-2 superconductors with second critical field $\mathrm{B}_{\mathrm{c} 2}>\mathrm{B}_{\mathrm{c}}$. On the other hand, $\mathrm{B}_{\mathrm{c} 2}=\Phi_{0} / 2 \xi \pi^{2}$ in which $\Phi_{0}$ is single magnetic flux quantum. As a result, we estimate $\xi=110 \mathrm{~nm}$, more than an order of magnitude lower compared to bulk Al. The penetration depth in this case is $\lambda=\xi_{0}\left(B_{c 2} / B_{c}\right)=300 \mathrm{~nm}$. Please note that according to textbooks the London penetration depth for bulk pure aluminum is $50 \mathrm{~nm}$. Corresponding relation $\lambda>\xi$, which is typical for a type-2 superconductor.

Two mechanisms responsible for changes of conductivity in the magnetic field are a change in the density of states of the superconductor due to depairing in the magnetic field [16] and changes in the conductivity in the region of Abrikosov's vortex, which can be directly measured by scanning tunnel spectroscopy [17].

In [16], the tunneling current was measured between a long narrow aluminum strip and the tunnel junction to copper. Side dimensions of the superconductor were significantly below London's penetration depth $\lambda$, and the normal magnetic field was uniform. For this case, tunneling current dependence on magnetic field corresponds to a simple formula for 
depairing energy $\Gamma / \Delta_{c}=\left(B / B_{0}\right)^{2}$, in which $B_{0} \sim 0.1 \mathrm{~T}$. In our case, the strip width is wider than $\lambda$, and the relation should be modified. However, if we compare the results, we can assume that at magnetic fields of 2.33 and $3.5 \mathrm{mT}$, the depairing mechanism is valid. This means that such fields are below the first critical field $\mathrm{B}_{\mathrm{c} 1}$.

For the field of $4.65 \mathrm{mT}$, conductivity at zero bias becomes three orders of magnitude larger than without the field. This means that the penetration of the magnetic field in the superconductor is completely different. In type-2 superconductors for magnetic fields above the first critical field $\mathrm{B}>\mathrm{B}_{\mathrm{c} 1}$, the magnetic field penetrates in the form of Abrikosov's vortices each carrying magnetic flux quantum $\Phi_{0}=2.07 \mathrm{mT} \times \mu \mathrm{m}^{2}$. The region of each tunnel junction with area $1.8 \times 1 \mu \mathrm{m}^{2}$ for the field of $4.65 \mathrm{mT}$ can contain approximately 4 vortices. In the case of coherence length $\xi \sim 110 \mathrm{~nm}$, the area of each vortex is approximately $0.04 \mu^{2}$, and they cover approximately $9 \%$ of the junction area. We can neglect interaction between vortices and assume that conductivity is proportional to the number of vortices. Even in the field of $2.33 \mathrm{mT}$, if the junction contains only two vortices, the conductivity should be two orders of magnitude above the measured value.

The simplest model that allows us to estimate the vortex conductivity is to assume that the vortex core locally forms a normal metal-insulator-normal metal (NIN) structure; see Figure 7 a. Its normalized conductivity will be $\pi \xi^{2} / S_{\text {NIS }} \approx 0.021$. For fields of 4.65 and $16.3 \mathrm{mT}$, we obtain the conductivity of 0.085 and 0.31 , which, taking into account the roughness of the model, can be considered close to the experimental values; see Figure 6. If it is just resistive shunting, the shape of conductivity curve will be as in Figure 6, dashed curves. Experimental curves are sufficiently different; they are not so flat, and additional currents make them similar to dependencies at higher temperatures. It is clear that in addition to simple shunting, there is an additional increase in conductivity at non-zero bias voltage.

The bias dependencies of dynamic conductivity in Figure 6 resemble conductivity dependencies at different electron temperatures. Additional overheating can be attributed to Joule heating of the whole structure by dc current via NIN shunting junctions. For example, let us estimate the heating of an SINIS with a normal electrode volume of $v=0.02 \mu \mathrm{m}^{3}$. Each vortex across the NIS area shunts it via the resistance of such NIN junction. Resistance can be obtained from the barrier parameter $R_{n} S=1 \mathrm{k} \Omega \times \mu \mathrm{m}^{2}$ and kernel area of $0.04 \mu \mathrm{m}^{2}$, which correspond to the resistance of such channel $R_{\text {kern }}=25 \mathrm{k} \Omega$. For one NIN junction with single-vortex resistance $R=25 \mathrm{k} \Omega$, the dissipated power is $\mathrm{P}=\mathrm{V}^{2} / \mathrm{R}$, half of which is dissipated in the absorber. The electron temperature at around half-gap bias voltage $\mathrm{V}=0.2 \mathrm{mV}$ for the normal metal absorber of the volume $v=0.02 \mu \mathrm{m}^{3}$ will be $T_{e}=\left(V^{2} / 2 R \Sigma v\right)^{0.2}$, i.e., approximately $T_{e}=0.6 \mathrm{~K}$. For presented IV curves at 0 , 7,17 , and $28 \mathrm{mT}$, we expect to have approximately $0,3,8$, and 14 vortices per square micrometer, so the corresponding electron temperatures at half-gap bias are 100, 400, 500, and $700 \mathrm{mK}$. Dependencies of the electron temperature on Joule heating via 1,3, and 9 NIN single normal channels are presented in Figure $7 \mathrm{~b}$. Such strong influence of the magnetic field and huge overheating is unacceptable for practical SINIS receivers, so avoiding the presence of vortices is a strong requirement for junction fabrication.

Here, it is necessary to notice that in SIS junctions with relatively thin $\mathrm{Al}$ electrodes, the same effect of magnetic field can lead to similar vortices and leakage bridges with resistance of the order of $25 \mathrm{k} \Omega$ and its fractions. If such junctions operate in detectors or parametric amplifiers at millikelvin temperatures, the overheating effect should also be taken into account.

Let us estimate whether overheating by the transport current can affect the dependence of conductivity on bias voltage in a case of an SIN thermometer. Let us consider for definiteness the case $\mathrm{B}=4.65 \mathrm{mT}$, voltage $0.01 \mathrm{~V}$ and current $0.7 \mu \mathrm{A}$. The power dissipated in the structure is $7 \mathrm{nW}$. The maximum effect will occur if all this power is dissipated in the normal electrodes. This power is dissipated into the phonon system of the electrodes and the gold wiring, the volume and area of which are enormous compared to those of the normal metal of the tunnel electrode. The total volume of all $100 \times 5$ gold film 
is $14 \times 100 \times 0.1 \times 100 \times 5=7 \times 10^{4} \mu \mathrm{m}^{3}$. Overheating due to Kapitza resistance can be neglected. We also neglect the temperature gradient along the wiring, assuming that its resistivity is the same as that of gold at room temperature. The estimate for the temperature difference between the regions of the film at $0.1 \mathrm{~K}$ is of the order of $10 \mathrm{mK}$. According to [17], for a gold film at temperatures below $0.5 \mathrm{~K}$, the electron-phonon interaction constant is $\Sigma=5-8 \times 10^{-9} \mathrm{~W} / \mu \mathrm{m}^{3} \times \mathrm{K}^{4}$. Thus, at $\mathrm{P}=7 \mathrm{nW}$ and $\mathrm{T}_{\text {substrate }}=0.1 \mathrm{~K}$, we have $7 \times 10^{-9}=$ $6.5 \times 10^{-9} \times 7 \times 10^{4} \times\left(\mathrm{T}^{4}-0.1^{4}\right)$ and $\mathrm{T}=0.104 \mathrm{~K}$. Thus, the change in the conductivity of a superconductor far from the vortex core remains 3-4 orders of magnitude smaller than for a normal metal, and such a change does not affect the total conductivity of the tunneling junction in the presence of a vortex structure.

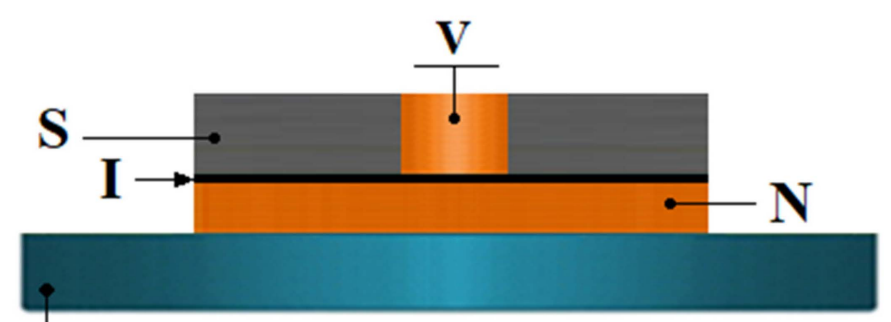

Si substrate

(a)

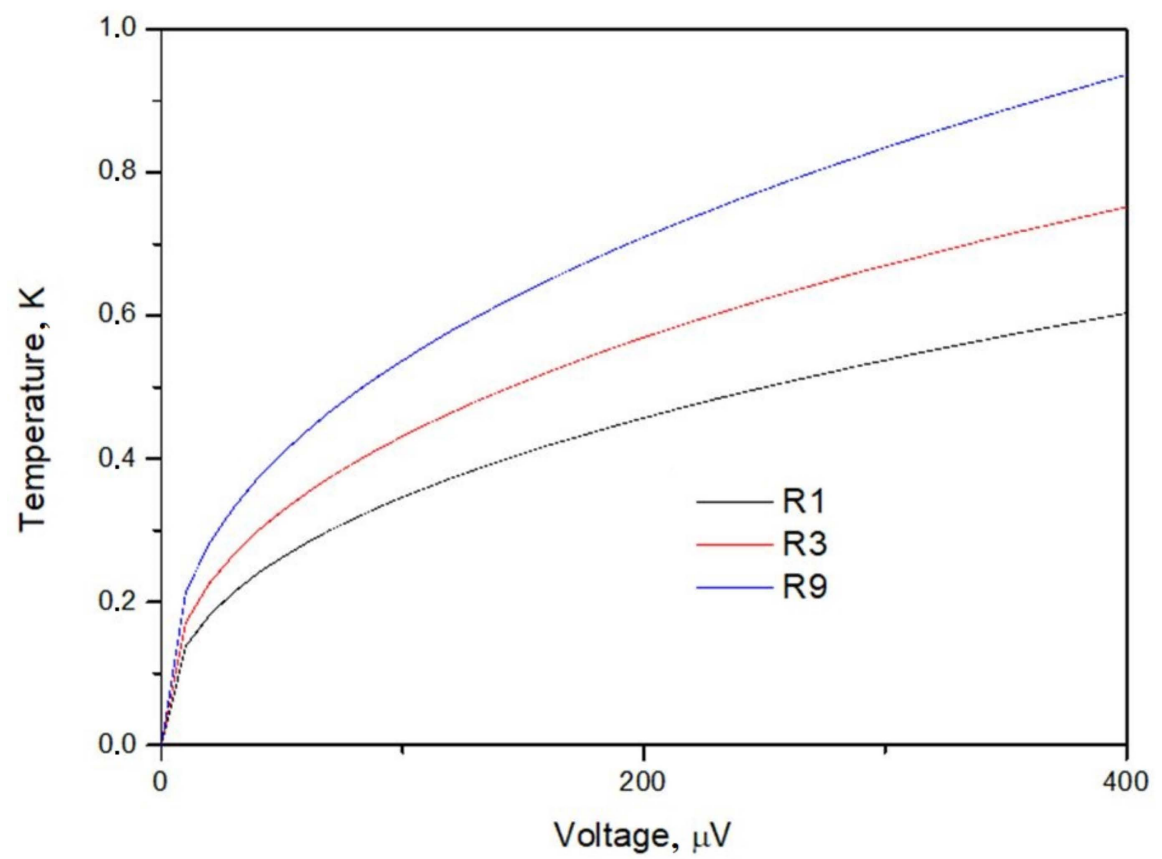

(b)

Figure 7. (a) Schematic view of NIS junction of $1 \times 1 \mu \mathrm{m}$ in size with $0.2 \mu \mathrm{m}$ flux kernel, here: Nnormal metal film; S-superconducting electrode; I-insulator; V—normal kern of single Abrikosov's Vortex forming NIN tunnel junction shunting NIS junction; (b) addition to electron temperature due to overheating through $1,3,9$ NIN junctions.

A more realistic model can be based on the results of studying vortices by scanning tunneling spectroscopy. In [18], it was found that the density of states normalized to the density in the middle of the vortex, corresponding to the normal state, at zero bias is described by the dependence

$$
\sigma(x, 0)=\sigma_{0}+\left(1-\sigma_{0}\right)\left(1-\tanh \left(\frac{x}{\xi}\right)\right)
$$


where $\sigma_{0}$-conductivity at $\mathrm{V}=0$ at long distance from the core of vortex. For the axially symmetric vortex, the full conductivity is $\sigma=\int \sigma(\mathrm{r}, 0) 2 \pi \mathrm{rdr}$ in the area of the radius significantly above $\xi$. The calculation shows that for $r>5 \times \xi$ (but below the junction area), the result is practically independent of $\xi$, if $\sigma_{0}<10^{-4}$, which is correct in our case. Due to the dirty aluminum value of $\xi=0.11 \mu \mathrm{m}$, according to (3), the conductivity for single vortex is $\sigma=0.031$. For 4 vortices, conductivity will be $\sigma=0.12$, which is close to the measured value of 0.09 . However, as measurements at 3.5 and $2.33 \mathrm{mT}$ show, partial penetration of the field in the edge regions of the film can sufficiently change the number of vortices in the area of the tunnel junction. Therefore, a self-consistent calculation of the field distribution is required.

It is rather difficult to describe the dependences $G(V)$ exactly. According to Figure 6, the conductivity maximum at the voltage around the energy gap $\mathrm{V}_{\Delta}$ is significantly smeared and shifted to lower voltages with the increase in the magnetic field. This means that the gap becomes coordinate-dependent and, on average, it decreases. Taking into account that this occurs at sizes comparable to $\xi$, such a calculation should take into account nonlocal effects.

\section{Discussion}

Aluminum-based superconducting junctions are often assumed as being made of type-1 superconductors, which can contradict experimental results. For thin granular films, it is possible to observe the penetration of the magnetic field, second critical field, enhanced critical current, and temperature. Such behavior of dirty films can be advantageous for increasing operation temperature and critical current, but leads to such drawbacks as additional overheating, noise, shunting of dynamic resistance, and a decrease in the sensitivity of various devices. To avoid vortex formation, the $\mathrm{Al}$ film thickness should be above the penetration depth of about $100 \mathrm{~nm}$ and be rather smooth. Grain size and surface roughness can be reduced by epitaxial growth on hot substrate, or evaporation on a cold substrate, obtained even by a Peltier cooler, down to $-27^{\circ} \mathrm{C}$, or better, down to $77 \mathrm{~K}$ by liquid nitrogen. The presence of Andreev current is another source of errors in the estimation of junction parameters based only on a single-electron tunneling mechanism for an ideal NIS junction. Taking into account both Abrikosov vortices and Andreev currents allows us to describe and predict IV characteristics of NIS junction-based structures with high accuracy.

\section{Conclusions}

The superconducting properties of Al films, tunnel NIS and SIS junctions and devices can be affected by the magnetic field, which suppresses resistance, and leads to the overheating and degradation of all the parameters of fabricated SINIS detectors and coolers, NIS thermometers, SIS Josephson junctions and SQUIDs. Improvement can be achieved by reducing the surface roughness and, at the same time, by increasing film thickness to over $150 \mathrm{~nm}$. Such films can be fabricated by cross-type lithography with thermal evaporation, or magnetron sputtering with double patterning. Penetration of a magnetic field normal to the surface in the form of Abrikosov vortices into granular aluminum films and the retention of superconductivity at fields higher than the critical value for bulk aluminum indicate that the films are type II superconductors. In this case, kernels of vortices form NIN nanocontacts with resistance comparable to quantum of resistance $h / 2 e^{2}=12 \mathrm{k} \Omega$, and containing a single flux of the magnetic field. Our experiments show that cooling even in a low magnetic field of approximately $1 \mathrm{mT}$ can lead to the suppression of the dynamic resistance of the junction, and it is important to provide a magnetic shield even in experiments with SINIS bolometers, contrary to the illusion that such devices are not affected by the magnetic field. 


\section{Patents}

1. Tarasov, M.; Gunbina, A.; Nagirnaya, D.; Fominsky, M. Method of Making Device with Thin-Film Tunnel Junctions. Patent RU2733330C1 2019, Russia.

2. Tarasov, M.; Gunbina, A.; Chekushkin, A.; Fominsky, M. Method of fabrication of thin-film tunnel junctions by means of separate lithography. Patent RU 2757762 priority from 30 March 2021, Russia.

Author Contributions: Conceptualization, V.E. and M.T.; methodology, V.E. and A.K.; validation, V.V., V.K. and A.K.; investigation, M.F. and A.C.; resources, V.K.; data curation, E.S.; writing-original draft preparation, M.T., V.E. and A.K.; writing—review and editing, M.T.; supervision, V.E.; project administration, V.K; fabrication, A.G. All authors have read and agreed to the published version of the manuscript.

Funding: The study was supported by a grant from the Russian Ministry of Science and Higher Education under grant no. 075-15-2021-990. This work was supported at Chalmers University of Technology by Swedish Research Council and EU Horizon 2020 program.

Acknowledgments: We thank S. Kraevsky (Carl Zeiss, Moscow, Russia) for performing AFM imaging.

Conflicts of Interest: The authors declare no conflict of interest.

\section{References}

1. Tarasov, M.A.; Kuzmin, L.S.; Kaurova, N.S. Thin multilayer aluminium structures for superconducting devices. Instrum. Exp. Tech. 2009, 52, 877-881. [CrossRef]

2. Tarasov, M.; Edelman, V. Effect of a magnetic field on the conductivity of superconductor-insulator-normal metal tunnel junctions. JETP Lett. 2015, 101, 740-743. [CrossRef]

3. Liu, Y.; Haviland, D.B.; Nease, B.; Goldman, A.M. Insulator-to-superconductor transition in ultrathin films. Phys. Rev. B 1993, 47, 5931-5946. [CrossRef]

4. Dolan, G.J. Offset mask for lift-off photoprocessing. Appl. Phys. Lett. 1977, 31, 337-339. [CrossRef]

5. Lecocq, F.; Pop, I.M.; Peng, Z.; Matei, I.; Crozes, T.; Fournier, T.; Naud, C.; Guichard, W.; Buisson, O. Junction fabrication by shadow evaporation without a suspended bridge. Nanotechnology 2011, 22, 1-5. [CrossRef] [PubMed]

6. Tarasov, M.; Gunbina, A.; Nagirnaya, D.; Fominsky, M. Method of Making Device with Thin-Film Tunnel. Junctions. Patent No. RU2733330C1, 1 October 2020.

7. Tarasov, M.; Gunbina, A.; Chekushkin, A.; Fominsky, M. Method of Fabrication of Thin-Film Tunnel Junctions by Means of Separate. Lithography. Patent No. RU2757762, 30 March 2021.

8. Gunbina, A.; Tarasov, M.; Fominsky, M.; Chekushkin, A.; Yusupov, R.; Nagirnaya, D. Fabrication of aluminium nanostructures for microwave detectors based on tunnel junctions. In Advances in Microelectronics Reviews; Sergey, Y.Y., Ed.; IFSA Publishing: Barcelona, Spain, 2021; Volume 3, pp. 183-212, ISBN 978-84-09-33338-7/978-84-09-33339-4.

9. Tarasov, M.; Gunbina, A.; Lemzyakov, S.; Nagirnaya, D.; Fominsky, M.; Chekushkin, A.; Koshelets, V.; Goldobin, E.; Kalaboukhov, A. Development of Josephson travelling-wave parametric amplifier based on aluminum SIS junctions. Phys. Sol. State 2021, 63, 1377-1381. [CrossRef]

10. Edelman, V.S. A dilution microcryostat-insert. Instrum. Exp. Tech. 2009, 52, 301. [CrossRef]

11. Hekking, F.W.J.; Nazarov, Y.V. Subgap conductivity of a superconductor-normal metal tunnel interface. Phys. Rev. B 1994, $49,6847$. [CrossRef] [PubMed]

12. Seliverstov, A.V.; Tarasov, M.A.; Edelman, V.S. The Andreev conductance in superconductor-insulator-normal metal structures. J. Exp. Theor. Phys. 2017, 124, 643-656. [CrossRef]

13. Dynes, R.C.; Narayanamurti, V.; Garno, J.P. Direct measurement of quasiparticle-lifetime broadening in a strong-coupled superconductor. Phys. Rev. Lett. 1978, 41, 1509. [CrossRef]

14. Nahum, M.; Eiles, N.M.; Martinis, J.M. Electronic refrigerator based on a normal-insulator-superconductor tunnel junction. Appl. Phys. Lett. 1994, 65, 3123-3125. [CrossRef]

15. Giaever, I.; Megerle, K. Study of superconductors by electron tunneling. Phys. Rev. 1961, 122, 1101-1111. [CrossRef]

16. Anthore, A.; Pothier, H.; Esteve, D. Density of states in a superconductor carrying a supercurrent. Phys. Rev. Let. 2003, 90, 127001. [CrossRef] [PubMed]

17. Shen, M. Low-Temperature Electron-Phonon Interaction in Disordered Metal Thin Films and Applications to Fast, Sensitive Submillimeter Photon Sources and Detectors. Ph.D. Thesis, Yale University, New Haven, CT, USA, December 2005. No. 3214299.

18. Eskildsen, M.R.; Kugler, M.; Levy, G.; Tanaka, S.; Jun, J.; Kazakov, S.M.; Karpinski, J.; Fischer, O. Scanning tunneling spectroscopy on single crystal $\mathrm{MgB}_{2}$. Physica C Supercond. 2003, 385, 169-176. [CrossRef] 